\title{
BASES DE HORMIGÓN PARA POSTES DE MADERA
}

Ing. Esp. Jorge Omar Sosa $\left(^{*}\right)$

INSTITUCIÓN EN LA QUE SE HA REALIZADO EL TRABAJO: Secretaría de Energía de Corrientes

\section{PROBLEMA}

\section{SOLUCIÓN PROPUESTA}

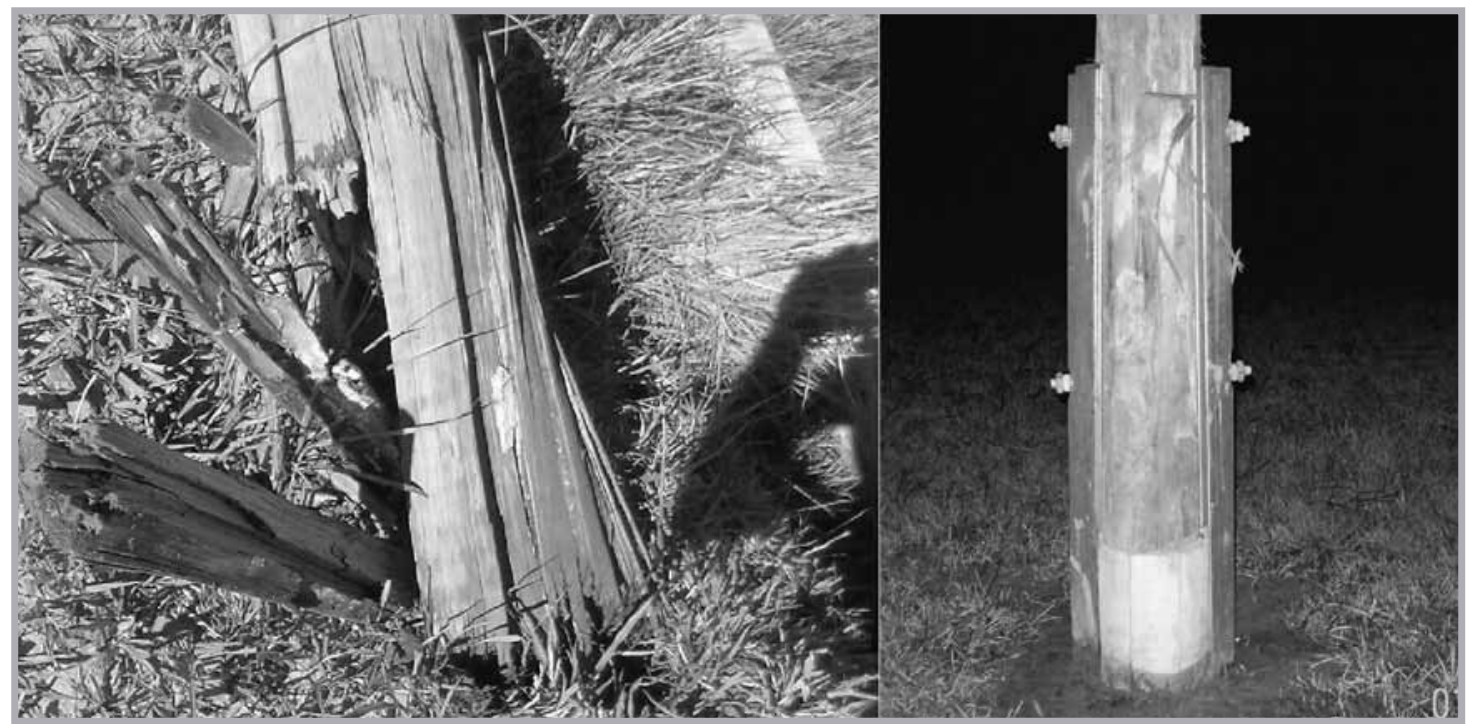

OBJETO DE ESTE TRABAJO: Evitar o demorar la putrefacción en la zona de transicion tierra-aire en la base de los postes

\section{ANTECEDENTES}

\section{ORIGEN DEL PROBLEMA}

Por ser los postes material orgánico, en su interacción con el suelo origina un proceso de descomposición en la madera por agentes biológicos. La mayoría de los cuales son hongos xilófagos que alteran la celulosa y lignina o ambos a la vez por la acción de diversas enzimas que segregan pudiendo producir la desintegración de las paredes celulares. En realidad los procesos se desarrollan con distintas velocidades en la zona donde se combinan materia orgánica(suelo), humedad y aire. Es aquí donde notamos que se crea un óvalo de descomposición que afecta al soporte, en mas o menos $30 / 35 \mathrm{~cm}$ por encima y por debajo del nivel de suelo.

(*) Prof. Adjunto D.S. Facena (Unne) - Direccion Postal: Mario Bandeo No62 (Resistencia -Chaco) -Cp3500 - Telefonos: 03624384964-03624435118 - E-mail: joromsosa@gmail.com, jor_omsosa@yahoo.com.ar 
SUELOS: Volviendo a la electrificación rural, en nuestra provincia los suelos son diversos, y en nuestras zonas rurales lo que caracteriza a muchos de nuestros caminos secundarios es que la extracción de material para levantar el mismo, produce zanjas que funcionan como escurrimiento del agua hacia las alcantarillas y por lo tanto afectan significativamente las posibilidades de una buena compactación, debido a que se acumula material orgánico en los lados y cuando a poca profundidad tenemos arcilla no filtra hacia abajo sino a los lados a través de los suelos areno/limosos superficiales, creando una flora diferente a la del campo después del alambrado.

Esto se da habitualmente de un solo lado del camino pero cuando este se estrecha porque en los caminos vecinales o algunas rutas provinciales avanzan con el alambrado o en algunos casos se saca suelo de ambos lados, tenemos un serio problema para implantar los soportes, por los problemas legales que se suscitan, las angulares que debemos colocar, etc.

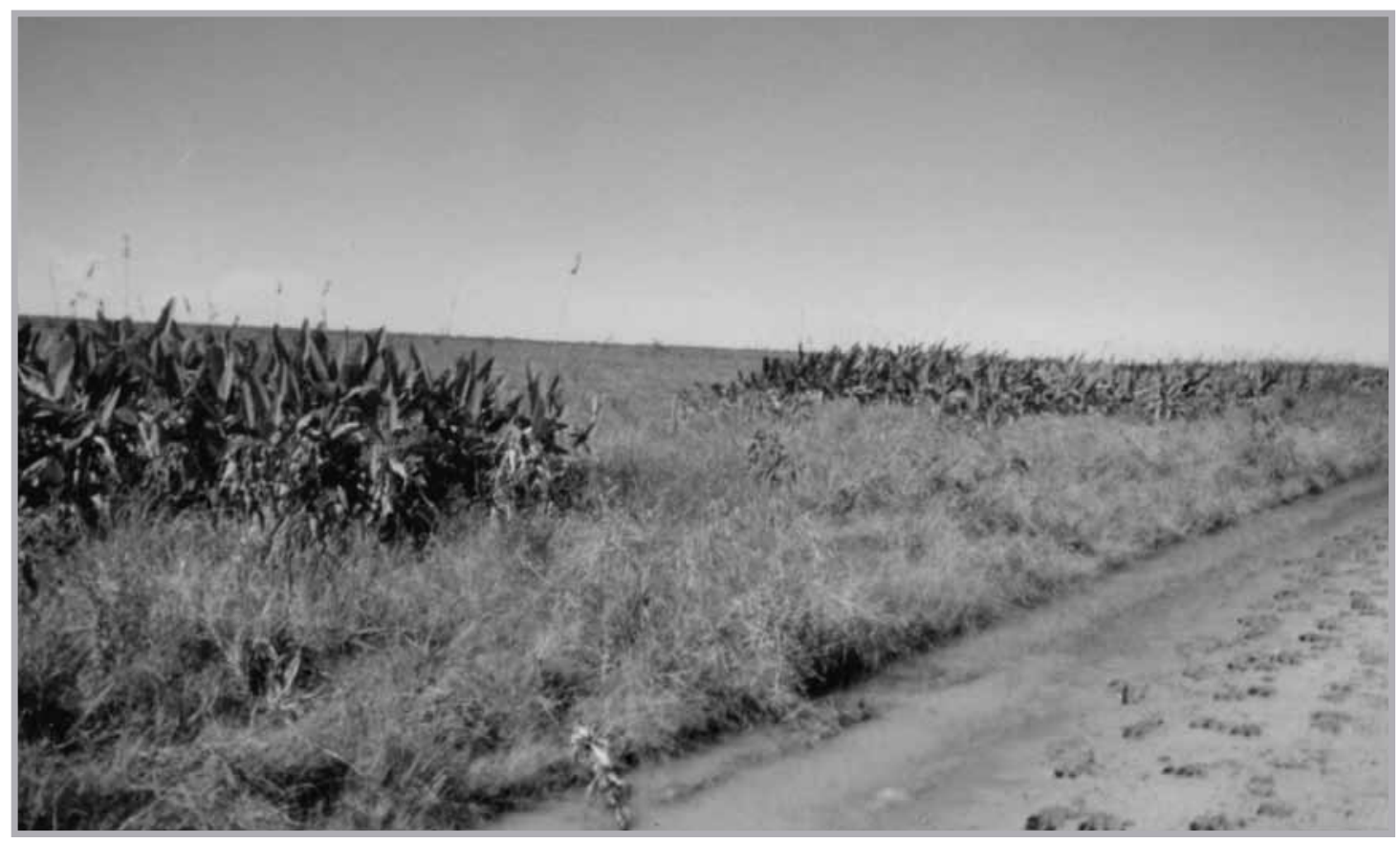

Vista de un camino rural típico

Un tema semántico a resolver es: ¿Cuando hablamos de terreno anegadizo y cuando de terrenos con agua permanente?, ya que en este último caso en los pliegos tradicionalmente se indica que es necesario colocar columnas de hormigón armado lo que encarece las obras por las razones antes señaladas. Las obras de electrificación rural generalmente tienen presupuestos ajustados y energizan zonas con problemas socio-económicos de larga data.

Si entendemos por terreno anegadizo aquel en que el agua solo està presente en la época de lluvias o como resultado de inundaciones extraordinarias en perìodos de pocas semanas (digamos 8 en el año máximo) teniendo seco el suelo mas del $80 \%$ del tiempo puede ser un criterio para definir que zonas tendrán un tipo constructivo u otro. 
Vialidad Nacional o Provincial nos exigen la utilización de una franja de $3 \mathrm{mts}$ paralela al alambrado lo que se vuelve inviable en muchos casos y dificulta el mantenimiento posterior. La inviabilidad es provocada porque en la mayoría de los casos el terreno para el camino se extrae del costado del camino, o de los dos costado creando una o dos zanjas antes del alambrado por donde debe ir la Línea de electrificación rural.

Además de lo señalado en el párrafo anterior, es muy común que cuando se cambia el alambrado perimetral de los campos se extienda en la zona prevista para camino público lo que nos complica aún más el acceso con equipo de izaje o transporte a la zona de trabajo.

ALTERNATIVAS DE BASES PARA SOPORTES Usando columnas de hormigón

Colocar una columna de hormigón de 8/R600 de 640kg con una base de hormigón que va a variar según el tipo de suelo, pero conservadoramente podemos estimar en $1.2 \mathrm{~m}^{3}$ va a requerir el uso:

a) Grùa apropiada 6/9Tnm de acuerdo al acceso

b) Tractor de tamaño adecuado para el ingreso de la grùa

c) Màquinas viales si se debe hacer un acceso a la zona de trabajo.

d) Acoplado playo y alzaprimas para su transporte

e) Hormigonera con motor de tamaño adecuado a la fundación a construir

f) Equipo de transporte de piedra, arena y cemento

g) Moldes, encofrados y herramientas menores.

h) Un estimado de 50/100 horas hombre para la fundación y la colocación de la columna, en general bastante impredecible ya que el tiempo y las condiciones del camino y acceso va a determinar los valores reales.

i) Estudio de suelo y un trabajo extra de ingeniería para hallar la mejor solución en los distintos casos que se presenten.

\section{SOLUCIONES SUGERIDAS PARA SUSPENSION}

Razones para adoptar este tipo constructivo:

a) El peso de la columna de hormigón corta ( 2 a 2,5ms) es manejable manualmente del orden de los $200 / 250 \mathrm{~kg}$

b) Con hoyadora se hace un hueco ligeramente superior al del tamaño de la columna a colocar y luego con pisones media luna y suelo cemento compacta formando un bloque 
compacto, se puede dar mayor longitud a la sección de columna en función de la calidad del suelo.

c) La unión se efectúa con perfiles MN556 para soportar el esfuerzo transversal que es el mayor debido al viento y por el que ceden los soportes de madera.

d) El costo de esta solución es mucho mas barata que cualquiera de las mencionadas, sobretodo si podemos utilizar alguna columna de hormigón en desuso con refuerzo adecuado

\section{SOLUCIONES ADOPTADAS PARA SUSPENSION}

De los ensayos surge que la solución combinada con crucetas, brazos MN40, cordones internos soldados, etc. Sería mas cara y no agregaría mas eficiencia. Se trató también de remediar la poca resistencia a la tracción en la cima demostrada en el ensayo con el agregado de una helicoide de varilla de hierro en las columnas de $\mathrm{H}^{\circ} \mathrm{A}^{\circ}$ cortas construídas.

Se deberían cortar los filetes de las varillas roscadas que excedan de 3 o 4 y puntearlos para que la vibración no los afloje(tienen además tuerca y contratuerca y arandela de presión).-

\section{SOLUCIONES SUGERIDAS ADOPTADAS PARA SUSPENSION}

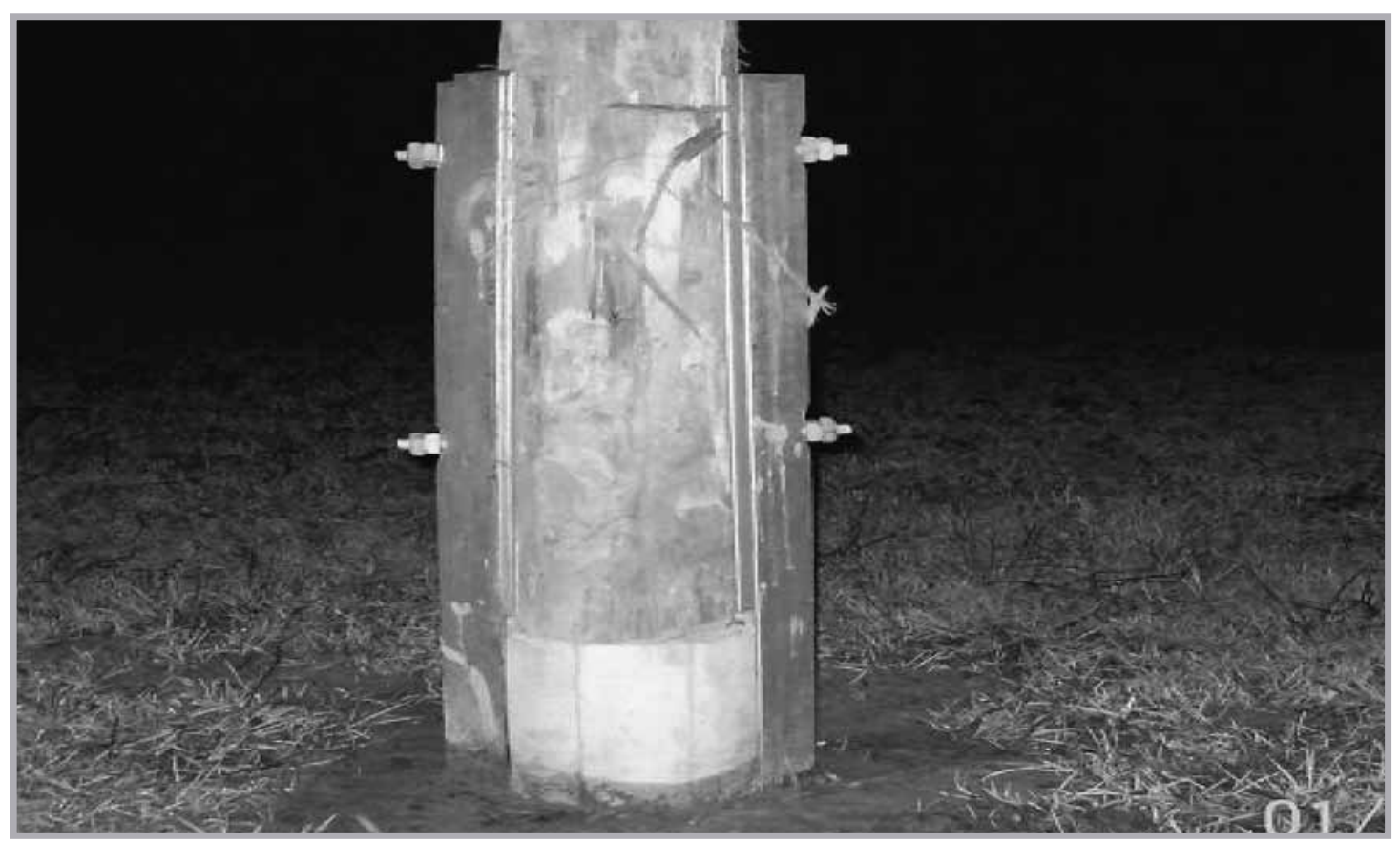

En este caso se tiene un diámetro un poco mayor de columna por lo que se coloca perfiles que suplementan la diferencia para que no haya esfuerzos de flexión al apretar las tuercas. 


\section{SOLUCIONES ADOPTADAS PARA ESTRUCURAS DOBLES}

En los dos casos se deberían cortar los filetes de las varillas roscadas que excedan de 3 o 4 y puntearlos para que la vibración no los afloje. No se ve el vínculo de hormigón armado entre las dos columnas cortas de hormigón. Siguen valiendo las razones señaladas para la solución de suspensión: peso, precio, posibilidad de trabajar sin equipo pesado. El vinculo entre los dos postes cortos de hormigón se puede hacer con un vinculo empleado en la Subestaciones donde se colocan los MN241 y los pararrayos, o armar in situ con un encofrado y los hierros adecuados.

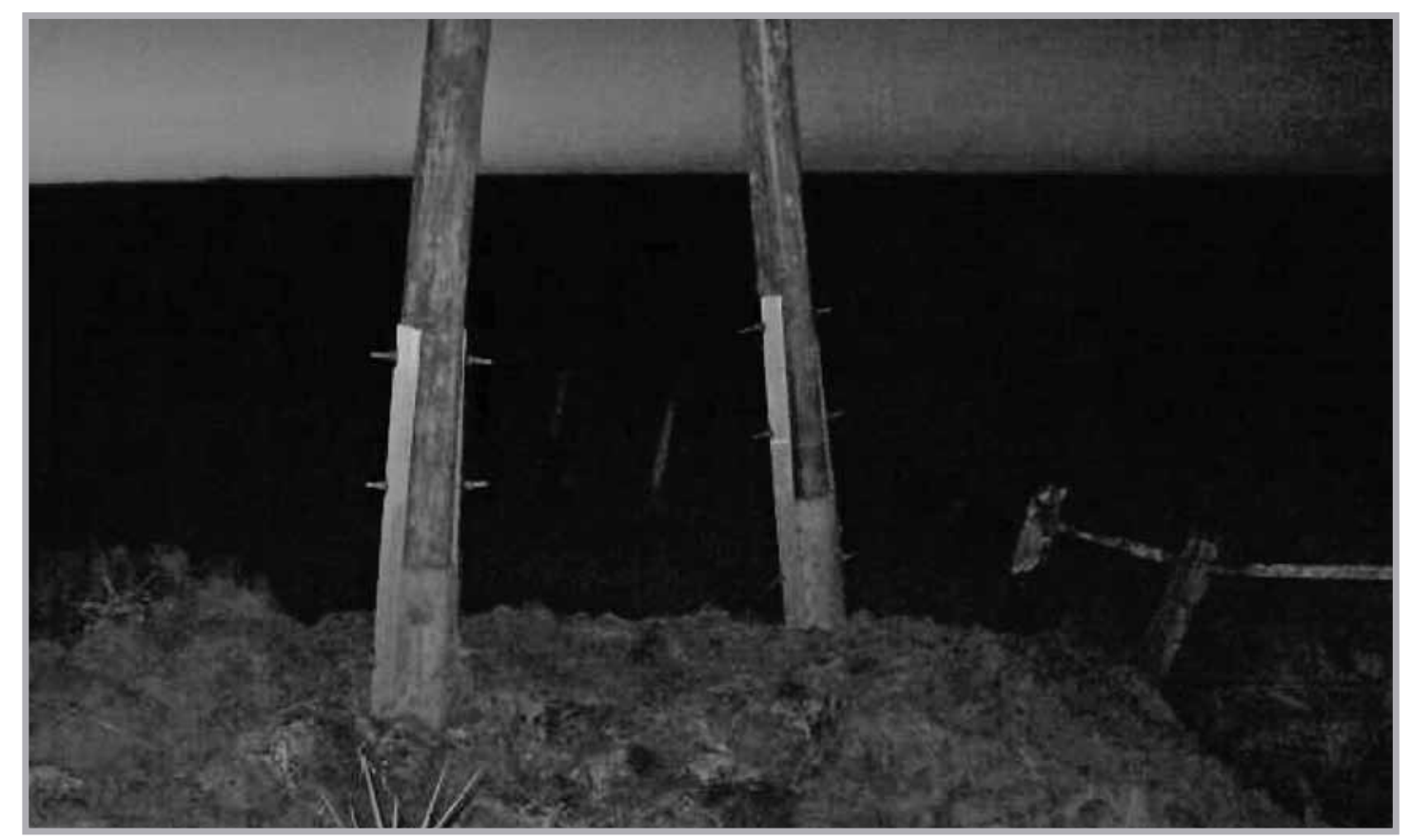

Verificacion por Sulzberger para postes o columnas empotrados sin fundación

Condiciòn de estabilidad : $\mathrm{Ms}+\mathrm{Mb}>=\mathrm{SxMv}$ donde

$$
\mathrm{Ms}=\mathrm{d}^{*} \mathrm{He}^{3 *} \mathrm{Ct}^{*} \mathrm{Tg} \rrbracket / 52,8 \text { y Mb }=\pi^{*} \mathrm{~d}^{*} \mathrm{dCb}{ }^{*} \operatorname{tg} \rrbracket / 64
$$

$$
\mathrm{Mv}=\mathrm{F}^{*}\left(\mathrm{Hl}+(2 / 3)^{*} \mathrm{He}\right)
$$

Verificación de los perfiles de alas iguales MN556: De acuerdo a las cargas presentes, que van a depender del vano y del cable a emplear se verifican los perfiles para los esfuerzos a la tracción y compresión que aparecen al ser sometidos a las cargas previstas.

ENSAYOS: Antes de su empleo se verificaron con las cargas previstas con un coeficiente de seguridad 3 , sin que se hayan constatado ningún tipo de fisuras en los postes de madera, daños en las columnas de $\mathrm{H}^{\circ} \mathrm{A}^{\circ}$ o en los perfiles 


\section{CONCLUSIONES}

Comparación de costos de colocación de Soportes de Suspensión equivalentes 8/ $\mathrm{R} 600$ y $\mathrm{Pm}$ 9/600/14 en zonas de agua permanente $\mathrm{o}$ anegadizas

\section{Para Soportes de Suspensión}

Variante 1: Columna de $\mathrm{H}^{\circ} \mathrm{A}^{\circ}$ con base de hormigón -

Costo estimado $\$ 8000$ a $\$ 12000$.-

Variante 2: Poste de madera corto con base de Columna de $\mathrm{H}^{\circ} \mathrm{A}^{\circ}$ de $2.5 \mathrm{~m}-$

Costo estimado $\$ 1200$ a $\$ 2500$.-

Las demás variantes en quebracho colorado, poste corto de madera dura, base de hormigón simple, etc. No se analizan en este caso.

Nota: Cabe acotar que en algunos casos la ejecución de soportes de suspensión es inviable por ser el costo mucho mayor al indicado en variante 1

\section{TRABAJOS EJECUTADOS EN ESTE TIPO CONSTRUCTIVO:}

Provincia De Corrientes.- Soportes colocados en: Ruta Provincial No $22-14 \mathrm{Km}$, Pje Albardones, Empedrado Limpio, Pje Galarcitas, Pago Poí, etc. En los últimos tres años a partir de la presentación de esta solución a la Secretaría de Energía de Corrientes.-

BIBLIOGRAFÍA SOBRE EL TEMA:

GAUDENCIO ZOPPETI - "Redes eléctricas de alta y baja tensión" 